\title{
Risk aversion, moral hazard, and the principal's loss ${ }^{\star}$
}

\author{
Hector Chade ${ }^{1}$ and Virginia N. Vera de Serio ${ }^{2}$ \\ 1 Department of Economics, Arizona State University, Main Campus PO Box 873806, \\ Tempe, AZ 85287-3806, USA (e-mail: hector.chade@asu.edu) \\ 2 Facultad de Ciencias Económicas, Universidad Nacional de Cuyo, 5500 Mendoza, ARGENTINA \\ (e-mail: vvera@fcemail.uncu.edu.ar)
}

Received: March 21, 2001; revised version: June 21, 2001

\begin{abstract}
Summary. In their seminal paper on the principal-agent model with moral hazard, Grossman and Hart (1983) show that if the agent's utility function is $U(I, a)=-e^{-k(I-a)}$, then the loss to the principal from being unable to observe the agent's action is increasing in the agent's degree of absolute risk aversion. Their proof is restricted to the case where the number of observable outcomes is equal to two, and it uses an argument that is specific to that case. In this note, we provide an alternative proof that generalizes their result to any (finite) number of outcomes.
\end{abstract}

Keywords and Phrases: Moral hazard, Principal-agent, Risk aversion.

\section{JEL Classification Numbers: D82.}

\section{Introduction}

Contracting problems with moral hazard have been widely studied in the economics literature in the last twenty years. The standard paradigm is the principalagent problem, where a risk neutral principal hires a risk averse agent to perform a certain task, but she cannot observe the action the agent takes. The contract is based on an observable stochastic outcome that depends on the agent's action. The basic problem for the principal in this situation is to design a compensation scheme that maximizes her expected profits.

\footnotetext{
* We thank Ed Schlee for his helpful comments. This work was partially supported by grant CD 30-99 from the Facultad de Ciencias Económicas-Universidad Nacional de Cuyo. 
It is well known that, compared to the first best case with observable actions, there is a nonnegative loss to the principal from being unable to observe the agent's action; i.e., her expected profits are smaller in the presence of moral hazard. However, this loss is zero if the agent is risk neutral.

In their seminal contribution, Grossman and Hart (1983) (henceforth GH) developed a useful two-step methodology to analyze the principal-agent problem when the number of outcomes is finite and the agent's preferences over income lotteries are independent of his actions. Among the numerous important issues addressed in their paper, they considered the following: since there is no incentive problem when the agent is risk neutral, but one exists when he is risk averse, does the loss to the principal increase when the agent becomes more risk averse? They immediately pointed out (GH p. 38) a difficulty in answering this question, namely, that if $U(a, I)$ is the agent's von Neumann-Morgenstern utility function that exhibits the aforementioned independence property, then an increasing concave transformation of this function (i.e., making the agent more risk averse) in general won't satisfy this property. In order to avoid this difficulty, they restricted attention to the case where the set of actions is a subset of the real line and $U(a, I)=-e^{-k(I-a)}, k>0 .{ }^{1}$ Assuming a finite number of actions and two outcomes, they were able to show that the loss to the principal is increasing in $k$; i.e., the more risk averse the agent is, the more costly is the incentive problem for the principal. The proof relies on an argument that is specific to the two-outcome case.

In this note, we provide an alternative proof that generalizes their result to any (finite) number of outcomes. ${ }^{2}$

\section{Preliminaries}

The incentive problem faced by the risk neutral principal is denoted by $(A, U, \bar{U}$, $q, \pi)$, where $A$ is the agent's action set, a finite subset of the real line; $U(a, I)=$ $-e^{-k(I-a)}, k>0$, is the agent's vonNeumann-Morgenstern utility function that depends on his income $I$ and his action $a ; \bar{U}=-e^{-k \alpha}$ is the agent's reservation utility, i.e. if he doesn't work for the principal he can obtain elsewhere a level of (certain) income $\alpha ; q=\left(q_{1}, \ldots, q_{n}\right)$ is the set of possible observable outcomes for the principal, $q_{1}<q_{2}<\ldots<q_{n} ; \pi(a)=\left(\pi_{1}(a), \ldots, \pi_{n}(a)\right)$ is the probability distribution over the outcomes if the agent chooses the action $a$, and it is assumed that $\pi_{i}(a)>0$ for every $a \in A$ and $i=1, \ldots, n$. An incentive scheme is simply

\footnotetext{
${ }^{1}$ This functional form has received considerable attention in empirical applications of the GH approach. See Haubrich (1994) and Haubrich and Popova (1998).

2 We recently became aware of a paper by Thiele and Wambach (1999) that analyzes how an increase in the agent's initial wealth affects the seriousness of the incentive problem. They provide a set of conditions under which the principal may prefer a 'poorer' agent. A change in the agent's initial wealth affects both his degree of risk aversion and the marginal rate of substitution between income and effort. Unlike Thiele and Wambach (1999), we (as GH) focus on a case with no wealth effects and with no changes in the marginal rate of substitution between income and effort as the agent becomes more risk averse.
} 
a vector $\left(I_{1}, \ldots, I_{n}\right)$, where $I_{i}$ is the agent's compensation when the outcome observed is $q_{i}$.

Let $B(a)=\sum_{i=1}^{n} q_{i} \pi_{i}(a)$ be the expected gross benefit for the principal when the agent takes an action $a$, and let $C(a, k)$ be the minimum expected cost for the principal if she wants to implement action $a$, when the agent's coefficient of absolute risk aversion is $k$. Formally, $C(a, k)$ is the value function of the problem

$$
\min _{I_{1}, \ldots, I_{n}} \sum_{i=1}^{n} \pi_{i}(a) I_{i}
$$

subject to

$$
\begin{aligned}
& -\sum_{i=1}^{n} e^{-k\left(I_{i}-a\right)} \pi_{i}(a) \geq-e^{-k \alpha} \\
& -\sum_{i=1}^{n} e^{-k\left(I_{i}-a\right)} \pi_{i}(a) \geq-\sum_{i=1}^{n} e^{-k\left(I_{i}-a^{\prime}\right)} \pi_{i}\left(a^{\prime}\right) \quad \forall a^{\prime} \in A,
\end{aligned}
$$

with the convention that $C(a, k)=\infty$ if the constraint set is empty.

$\mathrm{GH}$ introduced the following two-step procedure to solve for the optimal contract: first, for each $a \in A$, find $C(a, k)$; then, find the $a \in A$ that solves $\max _{a \in A} B(a)-C(a, k)$. They defined the loss to the principal from being unable to observe the agent's action as

$$
L(k)=\max _{a \in A}\left(B(a)-C_{F B}(a)\right)-\max _{a \in A}(B(a)-C(a, k)),
$$

where $C_{F B}(a)$ is the value function of problem (1) when the action is observable (first best case); it is easy to show that $C_{F B}(a)=a+\alpha$, which is independent of $k$.

The following result can be found in GH on p. 39:

Proposition 1 If $n=2$, then $L(k)$ is increasing in $k$.

In the next section, we provide a more general proof that holds for any $n \geq 2$. It makes use of the following result:

Lemma 1 Let $g(v)=-\ln (-v)+\frac{c}{v}, v<0, c>0$. Then $(i) g$ is strictly concave on the interval $(-2 c, 0)$ and strictly convex on $(-\infty,-2 c) ;($ ii $) g\left(\sum_{i=1}^{n} \pi_{i}(a) v_{i}\right) \geq$ $\sum_{i=1}^{n} \pi_{i}(a) g\left(v_{i}\right)$ if $\sum_{i=1}^{n} \pi_{i}(a) v_{i} \geq-c, \pi_{i}(a)>0$ and $v_{i}<0$ for every $i$.

Proof. See the Appendix.

\section{The result}

Rewrite (1) as follows:

$$
\max _{v_{1}, \ldots, v_{n}}-\sum_{i=1}^{n} \pi_{i}(a) h\left(v_{i}\right)
$$


subject to

$$
\begin{aligned}
e^{k a} \sum_{i=1}^{n} \pi_{i}(a) v_{i} & \geq \bar{U} \\
e^{k a} \sum_{i=1}^{n} \pi_{i}(a) v_{i} & \geq e^{k a^{\prime}} \sum_{i=1}^{n} \pi_{i}\left(a^{\prime}\right) v_{i} \quad \forall a^{\prime} \in A, \\
v_{i} & \in(-\infty, 0), \quad i=1, \ldots, n,
\end{aligned}
$$

where $h\left(v_{i}\right)=-\frac{\ln \left(-v_{i}\right)}{k}, v_{i}=-e^{-k I_{i}}$, and $\bar{U}=-e^{-k \alpha}$; obviously, the value function of this problem is $-C(a, k)$.

The following results will be needed:

Claim 1 If the constraint set is nonempty, then there exists a unique solution to problem (2).

Existence follows from Proposition 1 in GH, where they show that the constraint set is closed and one can artificially bound it. Since the objective function is strictly concave and the constraints are linear, the solution is unique.

Claim 2 The value function of problem (2) is differentiable in $k$, and the derivative is given by $-\frac{d C(a, k)}{d k}=\frac{\partial \mathscr{B}}{\partial k}$, where $\mathscr{B}$ is the Lagrangean of problem (2) evaluated at the optimum.

The conclusion follows because Claim 1 and the assumptions made ensure that the conditions of the Envelope Theorem in Milgrom (1999), Corollary 5, are satisfied.

Claim 3 If the constraint set of problem (2) is nonempty, then $e^{k a} \sum_{i=1}^{n} \pi_{i}(a) v_{i}=$ $\bar{U}$ at the optimum.

This follows from Proposition 2 in GH, which holds for the cost minimization problem for any given action for which a solution to problem (2) exists. ${ }^{3}$

We are now ready to prove the main result of the paper:

Proposition 2 For any $n \geq 2, L(k)$ is increasing in $k$.

Proof. As in GH, it suffices to show that $C(a, k)$ is increasing in $k$ for each $a \in A$ whenever $C(a, k)$ is finite. Since $-\frac{d C(a, k)}{d k}=\frac{\partial \mathscr{C}}{\partial k}$, we will prove that $\frac{\partial \mathscr{C}}{\partial k} \leq 0$.

Let $\lambda$ and $\mu_{a^{\prime}}, a^{\prime} \in A$, be the Lagrange multipliers associated with the individual rationality and incentive compatibility constraints of problem (2). By Claim 2, we can use the Envelope Theorem and obtain

$$
\begin{aligned}
\frac{\partial \mathscr{B}}{\partial k}= & -\frac{1}{k^{2}} \sum_{i=1}^{n} \pi_{i}(a) \ln \left(-v_{i}\right)+\lambda\left(a e^{k a} \sum_{i=1}^{n} \pi_{i}(a) v_{i}+\alpha \bar{U}\right)+ \\
& \sum_{a^{\prime} \in A} \mu_{a^{\prime}}\left(a e^{k a} \sum_{i=1}^{n} \pi_{i}(a) v_{i}-a^{\prime} e^{k a^{\prime}} \sum_{i=1}^{n} \pi_{i}\left(a^{\prime}\right) v_{i}\right) .
\end{aligned}
$$

\footnotetext{
${ }^{3}$ If it did not hold with equality, then the principal could offer $v_{i}(1+\epsilon), i=1, \ldots, n$ and $\epsilon>0$; this vector satisfies all the constraints and has a lower expected cost for the principal.
} 
Now, $\frac{\partial \mathscr{C}}{\partial v_{i}}=0$ is equivalent to

$$
-\frac{1}{k} \frac{\pi_{i}(a)}{v_{i}}=\lambda e^{k a} \pi_{i}(a)+\sum_{a^{\prime} \in A} \mu_{a^{\prime}}\left(e^{k a} \pi_{i}(a)-e^{k a^{\prime}} \pi_{i}\left(a^{\prime}\right)\right) .
$$

Multiplying both sides of (4) by $v_{i}$, adding over $i$, using Claim 3 and the complementary slackness conditions $\mu_{a^{\prime}} \frac{\partial \mathscr{C}}{\partial \mu_{a^{\prime}}}=0$ gives

$$
\lambda=\frac{e^{k \alpha}}{k} .
$$

Inserting this expression in (4) and adding over $i$ yields

$$
-\frac{1}{k} \sum_{i=1}^{n} \frac{\pi_{i}(a)}{v_{i}}-\frac{e^{k(\alpha+a)}}{k}=\sum_{a^{\prime} \in A} \mu_{a^{\prime}}\left(e^{k a}-e^{k a^{\prime}}\right) .
$$

$\mu_{a^{\prime}} \frac{\partial \mathscr{b}}{\partial \mu_{a^{\prime}}}=0$ and Claim 3 imply that for all $a^{\prime} \in A$

$$
\mu_{a^{\prime}} e^{k a^{\prime}} \sum_{i=1}^{n} \pi_{i}\left(a^{\prime}\right) v_{i}=\mu_{a^{\prime}} e^{k a} \sum_{i=1}^{n} \pi_{i}(a) v_{i}=\mu_{a^{\prime}} \bar{U}
$$

which allows us to rewrite (3) as follows:

$$
\frac{\partial \mathscr{D}}{\partial k}=-\frac{1}{k^{2}} \sum_{i=1}^{n} \pi_{i}(a) \ln \left(-v_{i}\right)-\frac{1}{k}(\alpha+a)+\sum_{a^{\prime} \in A} \mu_{a^{\prime}} \bar{U}\left(a-a^{\prime}\right) .
$$

Now, by convexity

$$
e^{k a^{\prime}}-e^{k a} \geq k e^{k a}\left(a^{\prime}-a\right)
$$

for all $a^{\prime} \in A$.

Since $\bar{U}<0$ and $\mu_{a^{\prime}} \geq 0$,

$$
k e^{k a} \mu_{a^{\prime}} \bar{U}\left(a-a^{\prime}\right) \leq \mu_{a^{\prime}} \bar{U}\left(e^{k a}-e^{k a^{\prime}}\right) .
$$

Adding over $a^{\prime}$ and using (5) yields

$$
\begin{aligned}
k e^{k a} \sum_{a^{\prime} \in A} \mu_{a^{\prime}} \bar{U}\left(a-a^{\prime}\right) & \leq \sum_{a^{\prime} \in A} \mu_{a^{\prime}} \bar{U}\left(e^{k a}-e^{k a^{\prime}}\right) \\
& =\bar{U}\left(-\frac{1}{k} \sum_{i=1}^{n} \frac{\pi_{i}(a)}{v_{i}}-\frac{e^{k(\alpha+a)}}{k}\right) \\
& =\frac{1}{k}\left(e^{k a}+e^{-k \alpha} \sum_{i=1}^{n} \frac{\pi_{i}(a)}{v_{i}}\right) .
\end{aligned}
$$

Therefore 


$$
\begin{aligned}
k^{2} \frac{\partial \mathscr{B}}{\partial k} & \leq \sum_{i=1}^{n} \pi_{i}(a)\left(-\ln \left(-v_{i}\right)+\frac{e^{-k(\alpha+a)}}{v_{i}}\right)-k(\alpha+a)+1 \\
& =\sum_{i=1}^{n} \pi_{i}(a) g\left(v_{i}\right)-k(\alpha+a)+1 \\
& \leq g\left(\sum_{i=1}^{n} \pi_{i}(a) v_{i}\right)-k(\alpha+a)+1,
\end{aligned}
$$

where the last two lines use the definition of $g$ and property (ii) of Lemma 1, respectively, with $c=e^{-k(\alpha+a)}$ and $\sum_{i=1}^{n} \pi_{i}(a) v_{i}=-c$, which follows by Claim 3.

But it is straightforward to show that $g\left(\sum_{i=1}^{n} \pi_{i}(a) v_{i}\right)=k(\alpha+a)-1$; hence $\frac{\partial \mathscr{C}}{\partial k} \leq 0$ and $\frac{d C(a, k)}{d k} \geq 0$, completing the proof of the proposition. ${ }^{4}$

\section{Appendix}

Proof of Lemma 1. (i) follows by straightforward differentiation. Observe that $g$ is (strictly) decreasing on the interval $(-c, 0)$ and (strictly) increasing on $(-\infty,-c)$; to prove (ii), we first show that, if $\beta y+(1-\beta) z \geq-c$ where $z<y<0$ and $0<\beta<1$, then

$$
g(\beta y+(1-\beta) z) \geq \beta g(y)+(1-\beta) g(z) .
$$

Notice that, if $z \geq-2 c$, then (6) follows from the first part of (i). Suppose $z<-2 c$; it is obvious that $y>\beta y+(1-\beta) z \geq-c>z$. Let $0 \leq \theta<1$ be such that

$$
\beta y+(1-\beta) z=\theta y+(1-\theta)(-c) .
$$

Algebraic manipulation yields

$$
\theta=1-\frac{(1-\beta)(y-z)}{y+c} .
$$

Since $z<-c$ and

$$
\beta=1-\frac{(1-\beta)(y-z)}{y-z},
$$

it follows that $\beta>\theta$.

\footnotetext{
${ }^{4}$ A careful inspection of the proof reveals that, except for the case where all the components of the vector $\left(v_{1}, v_{2}, \ldots, v_{n}\right)$ are equal, $C(a, k)$ is actually strictly increasing in $k$.
} 
Therefore

$$
\begin{aligned}
g(\beta y+(1-\beta) z) & =g(\theta y+(1-\theta)(-c)) \\
& \geq \theta g(y)+(1-\theta) g(-c) \\
& =\theta(g(y)-g(-c))+g(-c) \\
& \geq \beta(g(y)-g(-c))+g(-c) \\
& =\beta g(y)+(1-\beta) g(-c) \\
& \geq \beta g(y)+(1-\beta) g(z),
\end{aligned}
$$

where the first inequality uses (i), the second follows from $\beta>\theta$ and $g$ decreasing on $(-c, 0)$, and the last one uses the fact that $g$ is increasing on $(-\infty,-c)$.

We now complete the proof as follows. Suppose that $\sum_{i=1}^{n} \pi_{i}(a) v_{i} \geq-c$; it is obvious that $v_{i} \geq-c$ for at least one $i$. If $v_{i} \geq-2 c$ for every $i$, then from the first part of (i)

$$
g\left(\sum_{i=1}^{n} \pi_{i}(a) v_{i}\right) \geq \sum_{i=1}^{n} \pi_{i}(a) g\left(v_{i}\right) .
$$

If $v_{i}<-2 c$ for some $i$, let $\mathscr{T}_{1}=\left\{i: v_{i}<-c\right\}$ and $\mathscr{T}_{2}=\left\{i: v_{i} \geq-c\right\}$; these are nonempty sets. Consider

$$
\begin{aligned}
\beta & =\sum_{i \in \mathscr{T}_{2}} \pi_{i}(a), \\
y & =\sum_{i \in \mathscr{T}_{2}} \frac{\pi_{i}(a)}{\beta} v_{i}, \\
z & =\sum_{i \in \mathscr{T}_{1}} \frac{\pi_{i}(a)}{1-\beta} v_{i} .
\end{aligned}
$$

Notice that $0<\beta<1$ and

$$
\beta y+(1-\beta) z=\sum_{i=1}^{n} \pi_{i}(a) v_{i} ;
$$

moreover, $y>-c>z$ (this follows from the fact that $\sum_{i=1}^{n} \pi_{i}(a) v_{i} \geq-c$, $\pi_{i}(a)>0$ for every $i$, and $v_{i}<-2 c$ for some $i$ imply that $v_{i}>-c$ for some $\left.i \in \mathscr{T}_{2}\right)$.

As before, let $0 \leq \theta<\beta<1$, be such that $\beta y+(1-\beta) z=\theta y+(1-\theta)(-c)$. Following the same steps as in (7) we get

$$
\begin{aligned}
g\left(\sum_{i=1}^{n} \pi_{i}(a) v_{i}\right) & =g(\beta y+(1-\beta) z) \\
& \geq \beta g(y)+(1-\beta) g(-c) \\
& \geq \beta \sum_{i \in \mathscr{T}_{2}} \frac{\pi_{i}(a)}{\beta} g\left(v_{i}\right)+(1-\beta) \sum_{i \in \mathscr{T}_{1}} \frac{\pi_{i}(a)}{1-\beta} g(-c) \\
& \geq \sum_{i=1}^{n} \pi_{i}(a) g\left(v_{i}\right),
\end{aligned}
$$


where the second inequality uses (i) and $\sum_{i \in \mathscr{T}_{1}} \frac{\pi_{i}(a)}{1-\beta}=1$, and the last line follows because $g$ is increasing on $(-\infty,-c)$.

\section{References}

Grossman, S., Hart, O.: An analysis of the principal-agent problem. Econometrica 51, 7-45 (1983) Haubrich, J.: Risk aversion, performance, and the principal agent problem. Journal of Political Economy 102, 258-276 (1994)

Haubrich, J., Popova, I.: Executive compensation: a calibration approach. Economic Theory 12, 561-581 (1998)

Milgrom, P.: The envelope theorems. Mimeo, Department of Economics, Stanford University (1999)

Thiele, H., Wambach, A.: Wealth effects in the principal-agent model. Journal of Economic Theory 89, 247-260 (1999) 